\title{
Five years REACH: lessons learned and first experiences - an industry's view
}

\author{
Angelika Hanschmidt ${ }^{1 *}$, Michael Lulei ${ }^{1+}$ and Andrea Paetz ${ }^{2 \dagger}$
}

\begin{abstract}
Five years after the entry into force of the European chemical regulation REACH, the chemical industry has done much build-up work under difficult framework conditions, in order to implement REACH. Now it is decisive to benefit from lessons learned, simplify procedures and agree on solutions for existing problems - in a fair and transparent communication process between the stakeholders concerned and the competent authorities.
\end{abstract}

Results and conclusions: The REACH Regulation is highly complex, and its requirements need to be implemented step by step. Practical implementation is a learning process for everyone involved: companies and competent authorities alike. Up until now, implementation is working generally. Only first experiences are just being gathered on many aspects so that it is much too early for a valid evaluation.

Cost and workload for registrations are immense for the chemical industry. Therefore, now, the experiences from the first registration phases need to be used for simplifying the procedures and making them more efficient.

In the evaluation, the expectations of the competent authorities and of the companies need to be aligned with each other. Scientific issues are to be resolved. Here, it must be ensured that well-founded expert opinions find acceptance and that alternative assessment methods find their place. A fair and transparent communication between the competent authorities and the companies on existing points of criticism is a basic prerequisite for improvements. Studies show that the new extended safety data sheets under REACH are seen as overly comprehensive and unintelligible, both by those compiling them and by users. Therefore, forthcoming activities need to aim at simplifying the procedure and making it manageable also for smaller enterprises.

The authorisation procedure is increasingly being politically exploited. This should be adjusted in support of focussing on real risks and analysis of management options which should be open in its results and involve the industry in an early stage.

The chemical industry is working at high pressure on complying with its complex REACH obligations. It needs a stable environment, i.e. workable requirements and reliable planning. Fundamental changes to the Regulation would put at stake what has already been achieved.

Keywords: REACH; Chemical legislation; Registration; Evaluation; Authorisation; Supply chain communication; Extended safety data sheet

\section{Background}

Among all sectors of the economy, the German chemical industry is the most strongly impacted by the European chemical regulation $\mathrm{REACH}$. The companies manufacture a huge variety of special, innovative substances and products. As a key sector, the chemical industry is of outstanding importance for economic developments in Germany and in Europe. With its diverse range of products, the

\footnotetext{
* Correspondence: hanschmidt@vci.de

${ }^{\dagger}$ Equal contributors

'Verband der Chemischen Industrie e.V., Frankfurt 60329, Germany

Full list of author information is available at the end of the article
}

chemical industry makes essential contributions to health and environmental protection and also to climate protection and resource conservation.

The companies of the chemical industry need to comply with numerous legal provisions which ensure the safe import, manufacture, processing and use of chemical products. Thus, in a global comparison and particularly with the REACH Regulation, the by far highest requirements to the safety of chemicals are made in Europe. Furthermore, a number of specific rules are in place, regulating inter alia biocides, plant protection 
products, pharmaceuticals, materials coming into contact with food, toys, health assessment of construction products or substances in electrical and electronic equipment. In relation to the safe handling, use and disposal of chemicals, there are additionally comprehensive national laws on occupational health and safety, the safety of industrial installations and environmental protection. In consequence and overall, Germany has a very high degree of regulation of an enormous complexity. However, the very existence of large numbers of laws and regulations does not automatically ensure improvements in health and environmental protection. Such improvements can be brought about only where legislation is understandable and can be put into practice.

The stability of legal requirements is another basic prerequisite for a workable and efficient implementation. This applies in particular to the REACH Regulation which entered into force more than 5 years ago in 2007 and whose implementation has really begun only just now. The practical impacts of the REACH implementation in the first registration phases are gradually starting to penetrate the supply chains. First substances are subject to authorisation. The necessary implementation aids, tools and guidance documents have been newly developed; some of them are still being completed and have to prove their worth in practice. Now, more small and medium-sized enterprises need to submit registrations to the European Chemicals Agency (ECHA) by mid-2018, respectively.

All stakeholders have done much build-up work. The German chemical industry association VCI has intensively supported its member companies in these activities. Thus, in a recently published survey within a REACH Review study [1], the various forms of assistance from national and European associations were deemed particularly helpful.

The companies of the German chemical industry are making all efforts to implement $\mathrm{REACH}$ correctly and in due course. To succeed in these efforts, they need an environment which is reliable and enables long-term planning: with stable requirements and obligations. A discussion about changes to the text of the REACH Regulation or an entirely new legislative procedure would cause considerable uncertainty. Meanwhile, established and well-functioning procedures might be put into question, endangering further success in implementation. Regarding the significant problems arising in handling safety data sheets, the competent authorities and industry should jointly find workable solutions.

Publically available data on $\mathrm{REACH}$ implementation (e.g. from the chemical agency ECHA) and experiences gained within the chemical industry and in working groups of Verband der Chemischen Industrie e.V. were used, aiming at giving an industry's view on the status of REACH implementation until 31 December 2012.

\section{Major current tasks}

The REACH Regulation consists of the following core elements: registration, evaluation, authorisation and restriction as well as communication along the supply chain. ${ }^{\mathrm{b}}$

The following are the major current tasks for companies in REACH implementation:

- Preparing the registration of substances manufactured in volumes from 1 to 100 tonnes/year by 31 May 2018 after having registered substances from 100 to 1,000 tonnes/year by 31 May 2013 . Now, more small- and medium-sized enterprises need to register substances.

- Communication along the supply chain with the new extended safety data sheets (eSDS) which need to be passed on for registered substances.

Downstream users must, inter alia, check if their own uses are covered and pass on information to their customers.

- Moreover, the evaluation and the authorisation procedures have started. Here, additional information must be submitted where required, and applications for authorisation need to be prepared.

\section{Results and discussion \\ Registration}

By 2018, the companies need to stepwise register with ECHA all substances which are manufactured or imported in the European Union in volumes of at least 1 tonne/year. Depending on the volume and the hazards of a substance, comprehensive studies as well as chemical safety assessments and risk assessments are necessary for every use in the value chain. Furthermore, the companies are under the obligation to work together in the preparation of substance dossiers.

The companies of the chemical industry coped with these challenges with great commitment, an immense workload and strong personal efforts of their staff. By the end of 2010, they achieved the first interim goal of the REACH Regulation: the submission of dossiers for substances manufactured in volumes of at least 1,000 tonnes/year and for certain dangerous substances. The total workload in this phase was increased moreover by the need to notify substances to the Classification and Labelling Inventory. Now, the companies have worked at high pressure on the preparation of dossiers with the deadline of 31 May 2013.

Meanwhile, until end of May 2013, a total of 33,000 registration dossiers for 6,600 unique substances have already been submitted to ECHA (regular updates of registration statistics available in [2]). With just under $25 \%$, the by far largest share of the registration dossiers in the first registration phase comes from Germany (Figure 1). In the Substance Exchange Fora $(\mathrm{SIEFs})^{\mathrm{c}}$, a 


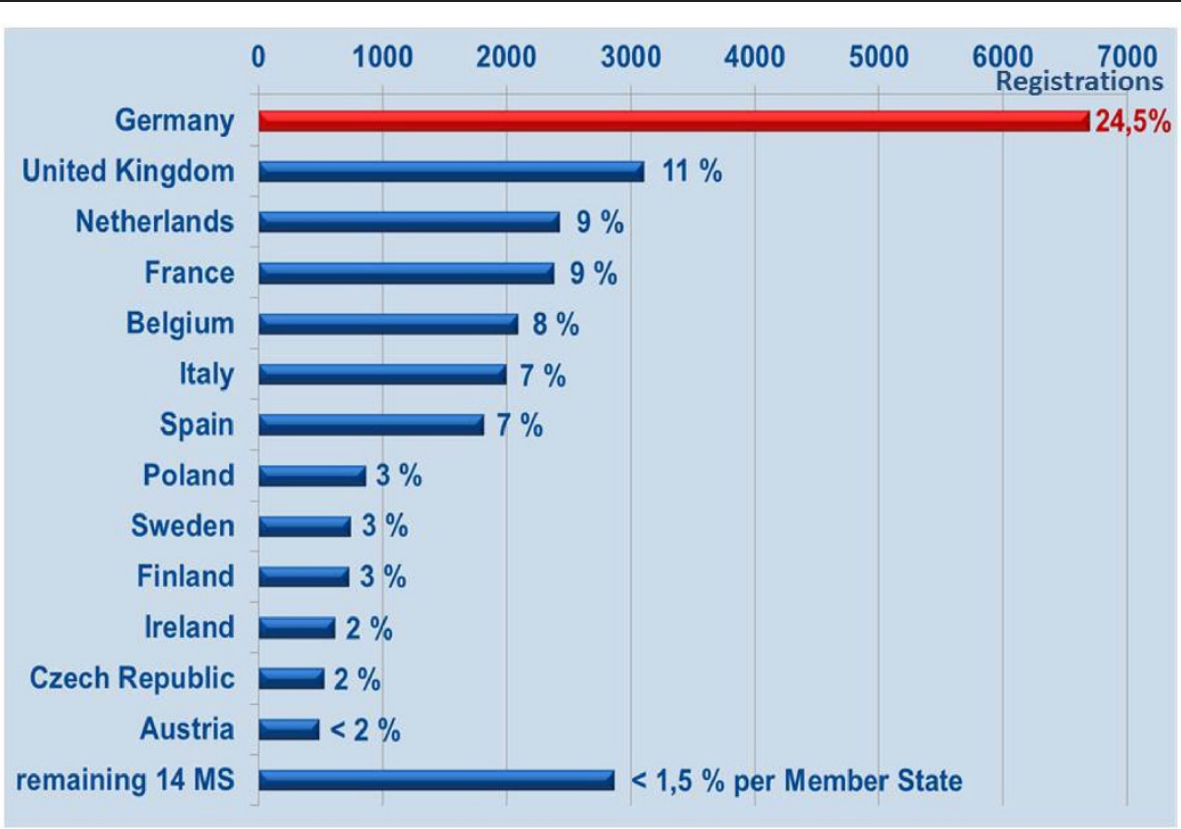

Figure 1 Share of the member states in registrations.

disproportionately large number of German companies are 'Lead Registrants', and in the first registration phase $40 \%$ of the lead dossiers were submitted by German companies [2].

The prescribed cooperation in SIEFs for the preparation of joint registration dossiers was new to many companies. This cooperation presupposes a well-coordinated, joint strategy between competitors. Moreover, the work took place in a difficult and changing environment:

- At the start of activities, the necessary information technology (IT) tools of ECHA (REACH-IT; International Uniform Chemical Information Database (IUCLID)) were still at a development stage. The first registration phase saw several version changes in the IUCLID software for dossier submission. This caused extra work on several occasions because the new versions needed to be integrated in the corporate IT systems, possibly with adaptations of workflows and staff getting familiar with changing framework conditions. ECHA was very late in providing aids for examining the completeness of dossiers and fees prior to submission. Another update of all IT tools was performed in July 2012.

- New interpretations of certain core requirements (e.g. definition of substance identity and of intermediates ${ }^{\mathrm{d}}$ ) rendered activities more difficult in the first registration phase as these new interpretations once more put into question some already-made decisions.
- Some ECHA guidance documents were elaborated in parallel to dossier preparation; others have been amended already several times. For example, this applies to the ECHA Guidance on intermediates that was last changed in late 2010 [3]. In various cases, the contributions from industry and legal opinions on specific issues were not taken into consideration sufficiently.

- Concepts and workflows for the cooperation in SIEFs and for preparing the registration documents needed to be developed from scratch. Supported by their associations (notably by the European Chemical Industry Council (Cefic) and VCI), the companies elaborated numerous guidance documents and, inter alia, model contracts for consortia and agreements with co-registrants. Elementary legal points needed to be clarified (e.g. regarding data sharing).

- Elaborating exposure scenarios for uses of dangerous substances within the chemical safety assessment was a totally new task, where associations and organisations (like Cefic and VCI) did build-up work.

- Additionally to the expert side, the organisation/ administration of consortia and SIEFs brought heavier workloads than had been expected.

- Updating the dossiers for registrations of new substances according to the 'old' chemicals act (Chemikaliengesetz) turned out particularly challenging; such substances are deemed registered under REACH. Relevant specific aspects had not been taken into account initially in the development 
of ECHA systems, and auxiliary structures became available only at a later time.

- Conversions/legal entity changes of companies are not unusual. However, transfers of registrations were included in the ECHA IT only after difficult discussions.

The VCI supported its member companies in this challenging phase - by providing up-to-date information, dealing with practical and legal questions in the VCI's working groups, offering information events and the Internet platform 'REACH umsetzen' which was created especially for REACH implementation.

In the second registration phase, registrants were benefiting from the earlier build-up work and from the experiences of existing registrants. Lessons should be learned from this. However, in the given system, the sharing of data and, in particular, the sharing of costs between competitors remain difficult tasks.

A stable environment is needed in further implementation so that the experiences gained in the build-up work during the initial phase can be used in implementation activities. A pragmatic course of action is even more important as a growing number of smaller businesses will be impacted in the registration phase to mid2018 , respectively. In view of this, the following actions are imperative:

- Companies need to have contact persons at ECHA. This can decisively contribute to not unnecessarily putting efforts in approaches which are subsequently rejected by the competent authorities.

- Inquiries prior to registration need to be processed faster.

- Amendments of guidance documents should focus on clarifications, examples from practice and more efficient practical strategies - and not on changes/ tightening of rules.

- Registrations should be gauged against the level of requirements applicable at the moment of submission.

- Updates of registrations need to be limited to the scope laid down in REACH; extra cost and work due to changes by ECHA to workflows, software and rules should be avoided.

- Adequate transitional periods need to be granted for changes that become necessary, e.g. because of amendments to the REACH Annexes.

- The use of methods intended under REACH to avoid animal testing, e.g. read-across and waiving, should not be rejected by ECHA unless for good reasons.

- Substance evaluation has just started. It is crucial that authorities ensure sufficient involvement of companies concerned in this process.

\section{Dissemination of information by ECHA}

According to the REACH Regulation, ECHA publishes several items of information (inter alia from the registration dossier) on the Internet [4]. This is intended to improve the knowledge on the properties and the risk potential of substances. The baseline study [5], which was performed on behalf of the EU Commission (Eurostat), already proves that this is the case. Meanwhile, ECHA makes publicly available a much wider range of information concerning, inter alia, the identity of the registrant and a more detailed breakdown of tonnage bands.

Moreover, after changes of publication rules by ECHA, the agency usually shifts dossier adaptation/update needs caused by these changes to the companies. One example is the confidential treatment of the company name for non-hazardous substances. In order to avert damage, companies were called upon to adapt their dossiers even though ECHA shares the position that there is no legal basis for publishing the company name for nonhazardous substances [6].

Publishing such information does not enhance the protection of health and environment. Instead, this provides competitors - especially those outside Europe with extensive and highly problematic insights in the portfolios of European companies, including their strategic orientation and planning. Thus, the competitiveness of European companies is impaired significantly. No other country of economic importance anywhere else in the world pursues such a far-reaching information policy which is detrimental to its own companies without any discernible reason.

The EU's Lisbon Strategy [7] adopted in 2000 wanted to make Europe the most competitive region worldwide. Against this backdrop and especially in view of the present economic and financial policy problems, a competitive industry should now be more than ever before in the interest of those responsible in Europe. Therefore, it is not understandable why the REACH Regulation is interpreted - without any need to do so - in such a way that it harms the competitiveness of own companies on the one hand without bringing any added benefit for health and environment on the other.

\section{IT tools of ECHA}

ECHA has already changed several times its rules for mandatory information in the IUCLID software for registrations and is currently planning further changes. This impacts dossier updates, too: it can become necessary to work on formerly accepted items of information beyond the actual changes for submission to function and/or for no confidential information to be published. For this reason, inter alia, the following points should 
be considered for REACH-IT and IUCLID to prevent unnecessary bureaucracy in the future:

- No further changes to mandatory fields, completing inevitable new fields should be optional and versions must be compatible.

- Data requirements are prescribed by the REACH Regulation and cannot be tightened by demands in the field of IT.

- More consideration needs to be given to know-how protection.

- Changes to chemical safety reports (e.g. for including an additional use) should be possible separately, i.e. without resubmitting the entire dossier.

- It should be examined whether simpler alternatives based on the IUCLID software can be made available for submitting registrations in 2018 .

\section{Evaluation}

The REACH Regulation includes both the evaluation of submitted registration dossiers by ECHA and substance evaluations led by the individual EU member states. First limited experiences are available from the dossier evaluation in $2011 / 2012$, but it seems too early and not really helpful to make general statements on the quality of registration dossiers already at the present stage. As is stated in REACH recital 21, the information yielded on substances through evaluation should be used in the first place by manufacturers and importers to manage the risks related to their substances.

Implementation in this phase is a learning process for companies and (competent) authorities - for both, the special requirements of a REACH dossier are new to most staff. Furthermore, the requirements for preparing a registration dossier are highly complex: in this exercise, ECHA guidance documents with several thousands of pages need to be taken into account and IT forms (IUCLID) with up to 15,000 data fields need to be completed. In this situation, it would be astonishing if there were no mistakes or conflicting views, especially in the initial phase.

For many points, workable approaches for practice need to develop gradually. In numerous cases, scientific issues remain to be clarified, too. The chemical industry accepts that ECHA demands justifications for expert opinions. Conversely, the competent authorities must ensure that well-founded expert opinions are accepted, alternative assessment methods (e.g. read-across and waiving) under $\mathrm{REACH}$ find their place and open scientific issues are clarified in cooperation between all stakeholders.

Individual stakeholders generally putting into question the quality of dossiers are rather counterproductive in this joint learning process. The 33,000 registration dossiers submitted to ECHA by European companies and representatives of non-European companies until end of May 2013 comprise many of good quality and, in a small number of isolated cases, clearly insufficient dossiers. Needless to say, insufficient registration dossiers are unacceptable. This is also in the interest of those companies who do whatever they can to prepare correct dossiers. In relevant cases, ECHA can subsequently make additional demands, and the enforcement authorities in the member states have adequate possibilities for sanctions. They can make additional demands locally and/or impose sanctions (for administrative offences and criminal acts). However, clearly insufficient and unacceptable registration dossiers should not be generalised and taken to refer to the industry in its entirety. Sweeping reproaches against 'industry' do not improve matters.

A fair and transparent dialogue between the industry and competent authorities is necessary, aligning the expectations of the competent authorities and their fulfilment by industry with each other. Feedback to the companies would be useful not only for subsequent additional demands but also on dossiers or parts of dossiers that were examined and accepted. This can contribute to more transparency in the evaluation procedure and to the forming of best practices. ECHA should name identified problems, preferably together with examples for improvement. For a more efficient and workable implementation, a pragmatic attitude should prevail over excessive formalities and perfectionism. Subsequent additional demands for information should be made only where they are relevant for a gain in safety.

For the time being, ECHA and the national authorities have a wealth of data and information on the individual substances which should be used first of all. The important point is to take the correct risk management measures for each substance. Instead of focusing on a purely formal adherence to high quality claims, the member states should primarily examine - based on the submitted information - whether the conclusions drawn in risk management are sufficient and acceptable. This should be done within the substance evaluation and in a pragmatic manner. Where gaps are identified, alternative risk management options should be equally examined before deciding for one measure. In particular, the following points should be considered for the future:

- Positive feedback to companies after dossier examinations without objections

- Highlighting of problematic points by ECHA together with best practice examples and alignment of ECHA's expectations with those of the impacted industry

- Transparent communication on ECHA's course of action in dossier screening 
- Possibility of contact persons

- Involvement of the impacted industry in substance evaluations

\section{Candidate list/authorisation procedure}

For substances with certain properties of very high concern (SVHC), the REACH Regulation gives the option of inclusion in the candidate list and possibly in Annex XIV (list of substances subject to authorisation). This brings information requirements for manufacturers, importers and suppliers of articles. Furthermore, an application for authorisation must be made for substances listed in Annex XIV of the REACH regulation [8]. After a transitional period, substances included in this list can be used only for expressly authorised uses for limited time spans.

The impression arises that the candidate list and the authorisation procedure are increasingly being politically exploited. For example, the Commission had set the target of a total of 136 substances being included in the candidate list [9] by the end of 2012. In order to meet this target, ECHA was retained to prepare inclusion dossiers for 37 substances within an extremely short time. Also, in December 2012, these substances, together with 17 substances identified in the regular process, were included in the candidate list - to allow ECHA and the Commission to declare target achievement with a total of 138 substances on the candidate list.

Here, the protection of health and environment was not in the fore. This was mainly about a political signal effect, as is highlighted by the following facts: the usually necessary analysis of uses and, based on this analysis, the choice of the best risk management method was largely not made. This is even more surprising, given that the authorisation procedure does not regulate, e.g. SVHC substances in imported articles. Consequently, articles containing these substances can be imported into the EU without authorisation becoming necessary. However, targeted restrictions would be the most effective way to regulate substances in imported articles (e.g. children's toys), thus protecting consumers.

This shows how easily the authorisation procedure and certain substances can become the objects of arbitrary political actions. Obviously, a certain 'target number' is to be achieved and hoped to attract much attention in the general public whilst improvements in safety are no longer striven for primarily. For realising the goals of the REACH Regulation, it would be more important for the candidate list/authorisation discussion to focus on substances where there are genuine problems and which cannot be adequately regulated by other pieces of legislation/measures, e.g. restrictions of critical uses or products. In any case, a risk management option (RMO) analysis is required prior to taking a decision on elaborating a dossier for inclusion of a substance in the candidate list, and the industry should already be involved in the RMO process in an early stage.

Moreover, the candidate list and the authorisation procedure stir great uncertainty among companies that use the listed substances in industrial processes and syntheses. This applies, e.g. for solvents or catalysts. According to the competent authorities, the latter substances are no longer deemed intermediates so that they can become subject to authorisation. Next, the concerned companies need to apply for authorisation, which involves much cost and work and an uncertain outcome. Failure to obtain authorisation for the catalyst or the solvent for just one out of several synthesis steps, e.g. in active substance synthesis for a pharmaceutical, would cause the entire synthesis process to collapse. Even if an authorisation is granted, it can be withdrawn at any time after having been reviewed.

High investments and, consequently, good planning security are prerequisites for constructing and operating industrial installations. The immense uncertainty due to Damocles' swords of 'candidate list' and 'authorisation procedure' makes many companies wonder whether investments in industrial plants in Europe can still be seen as a responsible decision - with potential impacts on the entire value chains.

Instead of exerting political pressure with the goal of driving up the number of substances on the candidate list or in the authorisation procedure, clear signals would be necessary instead, reassuring the actors that the mentioned uses will remain possible in the future. This would also be an important signal for Europe as an industry location and for strengthening the competitiveness of companies at this location.

\section{Communication along the supply chain}

$\mathrm{REACH}$ has challenging requirements for the communication between suppliers and customers on the safe use of substances and mixtures. The Regulation lays down a new format for an eSDS, i.e. a safety data sheet that needs to be extended by an annex with exposure scenarios. Following receipt of the eSDS, the users of chemicals need to check whether their substance uses were taken into account.

Different new contents of safety data sheets are to be generated, processed and communicated by mid-2015 or 2018. This means more mandatory information in the safety data sheet, new formats, new classification and labelling according to the CLP Regulation and new parameters - becoming available from registrations - for the risk assessment and the protection of humans and the environment (in particular DNELs, PNECs). The required exposure scenarios for substances need to be attached as an annex to the safety data sheet. Suppliers of mixtures, who do not need to prepare exposure 
scenarios themselves, can choose: either they can attach the relevant exposure scenarios as an annex to their safety data sheets or they can incorporate the relevant items of information in sections 1 to 16 .

In practice, it emerges that much build-up work needs to be done here, too. The actors are learning, inter alia, new use descriptions (ECHA Use Descriptor System [10]), and they are further developing them (e.g. additional specific environmental release categories). They are also preparing generic exposure scenarios. The VCI was early to provide assistance, inter alia, within its practical guide project [11].

Several studies mandated by the EU Commission within the REACH Review 2012 show that the safety data sheets are seen as overly comprehensive and unintelligible, both by those compiling them and by users. Whilst exposure scenarios - as part of the registration documentation - need to be prepared and understood by assessment experts, the safety data sheets are addressing a very wide range of different users (e.g. operations managers of chemical plants, small formulators of mixtures, users in other sectors and craftspeople).

Therefore, the competent authorities and industry should use this chance to jointly work on practical examples for the further strategy regarding the safety data sheet. This should be done in an open and solutionoriented manner. The Exchange Network on Exposure Scenarios (set up in late 2010) provides a platform for taking up such questions, developing a common understanding and best practices and triggering the necessary support activities in a targeted way.

For REACH to bring about the desired improvements in the safe use of chemicals in the supply chains, inter alia, the following factors are decisive:

- IT support tools need to be developed which facilitate the assessment activities and ease the burdens on experts in their routine work.

- The format of the safety data sheet needs to be reduced back to essential contents. The main goal: essential items of information for safe use in respect of occupational health and safety, protection of environment and consumers, and safety of industrial installations and in transport. Annex II to the REACH Regulation should be adapted accordingly, where necessary.

- Moreover, it should be examined to what extent a wider harmonisation of the format in chapters 1 to 16 and of exposure scenarios is possible. (An exchange format for exposure scenarios is already being developed). Furthermore, translations of standardised contents are necessary.

- An assessment of all identified uses along the entire supply chain and the life cycle approach make sense.
All the same, the question arises how, e.g. the burdens on craftspeople and consumer-related users can be eased without lowering the protection goals, and which simplifications are possible. Where important items of information are lost in the flood of information, intelligent courses of action should be identified and coordinated between the competent authorities and industry. The overarching goals of REACH need to be respected whilst reducing bureaucratic obstacles. In this regard, strategies, e.g. like that of Berufsgenossenschaft der Bauwirtschaft (BG BAU; employers' liability insurance of the construction industry), make interesting starting points; the BG BAU strategy is supported by the Federal

Environment Ministry and the Federal Institute for Occupational Safety and Health. Generic exposure scenarios are another example.

- REACH enforcement should avoid approaches which give rise to a purely formal fulfilment of obligations. Instead, the building of know-how for preparing good safety data sheets should be actively supported.

In the next steps, it is of primary importance to simplify the course of action for the safety data sheet and to make matters manageable also for SMEs - instead of further increasing the number of guidance.

\section{Conclusions}

The REACH Regulation is highly complex, and its requirements need to be implemented step by step. Practical implementation is a learning process for everyone involved: companies and competent authorities alike. Up until now, implementation is working generally. Only first experiences are just being gathered on many aspects so that it is much too early for a valid evaluation.

Cost and workload for registrations are immense for the chemical industry. Therefore, the experiences from the first registration phases now need to be used for simplifying the procedures and making them more efficient. ${ }^{\mathrm{e}}$

In the evaluation, the expectations of the competent authorities and of the companies need to be aligned with each other. Scientific issues are to be resolved. Here, it must be ensured that well-founded expert opinions find acceptance and that alternative assessment methods find their place, as provided by REACH. A fair and transparent communication between the competent authorities and the companies on existing points of criticism is a basic prerequisite for improvements.

Studies show that the new eSDS under REACH are seen as overly comprehensive and unintelligible, both by those 
compiling them and by users. For this reason, forthcoming activities of public authorities and companies need to aim at simplifying the procedure and making it manageable also for smaller enterprises.

The authorisation procedure is increasingly being politically exploited. This should be adjusted in support of focussing on real risks and an analysis of management options which should be open in its results and involve the industry in an early stage.

Supported by VCI, the chemical industry is working at high pressure on complying with its complex REACH obligations. For this purpose, the chemical industry needs a stable environment, i.e. workable requirements and reliable planning. Against this backdrop, fundamental changes to the Regulation would put at stake what has already been achieved.

Practical proposals for improvements are brought forward in this text. Furthermore, the chemical industry will also contribute its experiences into the discussion about the REACH Review.

\section{Endnotes}

${ }^{\mathrm{a}} \mathrm{REACH}$ legal text - weblinks to official text, amendments and implementing legislation in Official Journal of the European Union and consolidated version offered by the European Commission (http://ec.europa.eu/enterprise/ sectors/chemicals/documents/reach/index_en.htm\#h2-1).

${ }^{\mathrm{b}} \mathrm{A}$ compact overview of the functioning and the requirements of REACH is given, e.g. by the VCI in chemie report spezial: Registrierung 2013 - Starten Sie jetzt! (https://www. vci.de/Downloads/Publikation/chemie-report/cr2011_mai_ spezial.pdf).

${ }^{\mathrm{c}}$ Substance Information Exchange Forum (SIEF). All potential registrants of the same substance are by REACH definition members of a SIEF and shall share substance data, agree on classification and labelling and do joint submissions of registration dossiers.

${ }^{\mathrm{d}}$ For interpretations of REACH definitions by authorities, see guidelines of the European Chemicals Agency (http://echa.europa.eu/guidance-documents/guidance-onreach).

${ }^{\mathrm{e}} \mathrm{Some}$ figures on costs and workload linked to registrations under REACH Regulation are available from the CSES study on the functioning of the chemical market (see [1]); data for Germany have been assessed in a project by the German Nationaler Normenkontrollrat together with Bundesministerium für Umwelt, Naturschutz und Reaktorsicherheit, Bundesanstalt für Arbeitsschutz und Arbeitsmedizin, Umweltbundesamt, Verband der Chemischen Industrie; November 2012. (http://www.normenkontrollrat.bund. de/Webs/NKR/Content/DE/Publikationen/2012_11_30_ abschlussbericht_reach.pdf?_blob=publicationFile\&v=2).

\section{Abbreviations}

Cefic: European Chemical Industry Council; eSDS: Extended safety data sheet; REACH: Regulation (EC) No 1907/2006 concerning the Registration Evaluation, Authorisation and Restriction of Chemicals; RMO: Risk management option; SIEF: Substance Information Exchange Forum; SVHC: Substance of very high concern; VCl: Verband der Chemischen Industrie e.V.

\section{Competing interests}

The authors declare that they have no competing interests.

\section{Authors' contributions}

All authors - AH, ML, AP - contributed to all parts of this publication. All authors read and approved the final manuscript.

\section{Author details}

${ }^{1}$ Verband der Chemischen Industrie e.V., Frankfurt 60329, Germany. ${ }^{2}$ Bayer AG, Leverkusen 51368, Germany.

Received: 21 May 2013 Accepted: 31 May 2013

Published: 25 July 2013

\section{References}

1. Centre for Strategy \& Evaluation Services (CSES): Interim Evaluation: functioning of the European chemical market after the introduction of REACH. http://ec.europa.eu/enterprise/sectors/chemicals/documents/reach/ review2012/chemical_market_en.htm.

2. European Chemicals Agency (ECHA): Registration statistics from 31 May 2013. http://echa.europa.eu/information-on-chemicals/registration-statistics.

3. European Chemicals Agency (ECHA): Guidance on intermediates, Appendix 4 (Definition of Intermediates as agreed by Commission, Member States and ECHA on 4 May 2010). http://echa.europa.eu/documents/10162/13632/ intermediates en.pdf.

4. European Chemicals Agency (ECHA): Registered substances database. http:// echa.europa.eu/web/guest/information-on-chemicals/registered-substances.

5. European Commission - Eurostat: The REACH baseline study - 5 years update 2012. http://ec.europa.eu/enterprise/sectors/chemicals/documents/reach/ review2012/baseline_en.htm.

6. European Chemicals Agency (ECHA): Questions and answers - dissemination and confidentiality claims of safety data sheet information in IUCLID 5.4, version 1.2. http://echa.europa.eu/documents/10162/13651/ questions_and_answers_sds_info_dissemination_en.pdf.

7. European Council: Presidency conclusions, Lisbon European Council 23 and 24 March 2000. http://www.consilium.europa.eu/uedocs/cms_data/docs/ pressdata/en/ec/00100-r1.en0.htm.

8. European Chemicals Agency (ECHA): Addressing chemicals of concern: authorization list. Available [http://echa.europa.eu/web/guest/addressingchemicals-of-concern/authorisation/recommendation-for-inclusion-in-theauthorisation-list/authorisation-list]

9. European Chemicals Agency (ECHA): Candidate list of substances of very high concern for authorisation: published in accordance with Article 59(10) of the REACH Regulation. http://echa.europa.eu/web/guest/candidate-list-table.

10. European Chemicals Agency (ECHA): Guidance on information requirements and chemical safety assessment, chapter R.12: use descriptor system. http://echa. europa.eu/documents/10162/13632/information_requirements_r12_en.pdf.

11. Verband der Chemischen Industrie e.V. (VCI), The European Chemical Industry Council (CEFIC): REACH practical guide on exposure assessment and supply chain communication. http://www.vci.de/Themen/ Chemikaliensicherheit/REACH/Seiten/REACH-Praxisfuehrer.aspx.

doi:10.1186/2190-4715-25-19

Cite this article as: Hanschmidt et al:: Five years REACH: lessons learned and first experiences - an industry's view. Environmental Sciences Europe 2013 25:19. 